\title{
OPEN Dynamic changes in marital status and survival in women with breast cancer: a population-based study
}

\author{
Wu Ding ${ }^{1,3,5 凶}$, Guodong Ruan ${ }^{1,5}$, Yingli Lin ${ }^{4}$, Jianming Zhu ${ }^{1}$, Chuanjian Tu ${ }^{2,3}$ \& Zhian Li ${ }^{1,3}$
}

Marital status proved to be an independent prognostic factor for survival in patients with breast cancer. We therefore strove to explore the impact of dynamic changes in marital status on the prognosis of breast cancer patients. We selected patients meeting the eligibility criteria from the Surveillance, Epidemiology, and End Results cancer database. We then used multivariate Cox proportional hazard regression model to analyze the effect of dynamic changes in marital status on the prognosis of overall survival (OS) and breast cancer-specific special survival (BCSS). Compared with the patients in the Single-Single group and the divorced/separated/widowed-divorced/separated/ widowed (DSW-DSW) group, patients in the Married-Married group were significantly associated with better BCSS (HR 1.13, 95\% Cl: 1.03-1.19, $P<0.001$; HR 1.19, 95\% Cl: 1.14-1.25, $P<0.001$, respectively) and OS (HR 1.25, 95\% Cl: 1.20-1.30, $P<0.001 ; \mathrm{HR} 1.49,95 \% \mathrm{Cl}: 1.45-1.54, P<0.001$, respectively). In contrast to the DSW-DSW group, the Single-Single group and the DSW-Married group showed similar BCSS (HR 0.98, 95\% Cl: 0.92-1.05, $P=0.660 ; \mathrm{HR} 1.06,95 \% \mathrm{Cl}$ : 0.97-1.15, $P=0.193$, respectively) but better OS (HR 1.14, 95\% Cl: $1.09-1.19, P<0.001 ; \mathrm{HR} 1.32,95 \% \mathrm{Cl}: 1.25-$ $1.40, P<0.001$, respectively). Compared with the Single-Single group, the Single-Married group showed significantly better BCSS (HR 1.21, 95\% Cl: 1.07-1.36, $P=0.003$ ) but no difference in OS (HR $1.08,95 \% \mathrm{Cl}: 0.98-1.18, P=0.102)$; In contrast to the Married-DSW group, the Married-Married group exhibited better BCSS (HR 1.11, 95\% Cl: 1.05-1.18, $P<0.001$ ) and OS (HR 1.27, 95\% Cl: 1.22-1.32, $P<0.001$ ). Our study demonstrated that, regardless of their previous marital status, married patients had a better prognosis than unmarried patients. Moreover, single patients obtained better survival outcomes than DSW patients. Therefore, it is necessary to proactively provide single and DSW individuals with appropriate social and psychological support that would benefit them.

Breast cancer is the most frequently diagnosed cancer and the second leading cause of cancer-related deaths in women in the Western world ${ }^{1}$. As a systemic disease, the formation of breast cancer is the result of complex interactions between physiological and psychosocial factors ${ }^{2}$. Marital status, as one of the most important forms of social relations, influences those factors of breast cancer patients. A growing body of evidence suggests that marital status acts as an independent prognostic factor for survival in patients who experience breast cancer, and the mortality of breast cancer is higher in unmarried patients ${ }^{3-9}$.

Previous studies have confirmed that married patients can get more mental and financial support, have better compliance, which can help them to be diagnosed at an early stage and get more appropriate treatments, ultimately prolonging their survival ${ }^{10-13}$. Nevertheless, those researches provide information on marital status only from the time points of recently diagnosed breast cancer which may not reflect the dynamic changes in long-term survival populations or account for marital status changes before or after the cancer diagnosis. Moreover, further analysis of marital status changes during the period of breast cancer, which may reveal the potential mechanism of marital status affecting the prognosis of breast cancer, is also neglected.

The Surveillance, Epidemiology, and End Results (SEER) cancer database includes research data from 18 different population-based cancer registries which have been widely used for this kind of study that explores the relation between marital status and breast cancer survival rates among patients ${ }^{3,7-9,14}$. In view of the current research situation, we selected the data from SEER database and conducted this analysis to explore the

${ }^{1}$ Department of Oncological Surgery, Shaoxing Second Hospital, Shaoxing, China. ${ }^{2}$ Department of Neurosurgery, Shaoxing Central Hospital, Shaoxing, China. ${ }^{3}$ Department of Clinical Medicine, Shaoxing University School of Medicine, Shaoxing, China. ${ }^{4}$ Department of Early Childhood Education, Shaoxing Vocational and Technical College, Shaoxing, China. ${ }^{5}$ These authors contributed equally: Wu Ding and Guodong Ruan. ${ }^{\circledR}$ email: wuding0320@163.com 


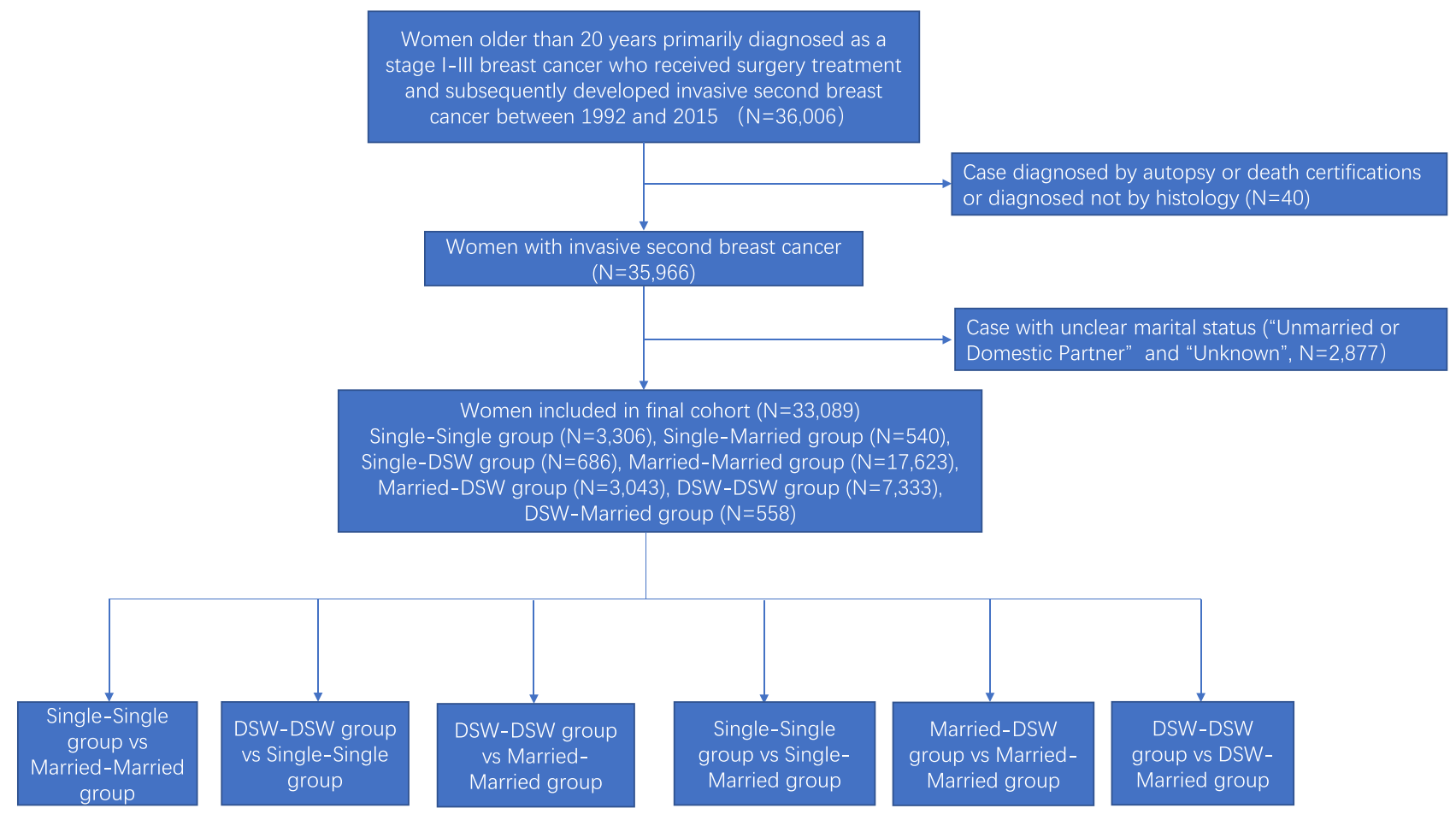

Figure 1. Flow diagram of patient population.

relationship between dynamic changes in marital status and overall survival (OS) or breast cancer-specific survival (BCSS).

\section{Materials and methods}

Patient selection and data extraction. To estimate the correlation between the dynamic changes in marital status and BCSS or OS in breast cancer patients, we used SEER ${ }^{\star}$ Stat 8.3.6 software to extract relevant information, including patient identification, year of diagnosis, age at diagnosis, race/ethnicity, marital status, insurance, 6th tumour-node-metastasis (TNM) staging classification, histology type, nuclear grade, oestrogen receptor (ER) status, progesterone receptor (PR) status, surgery, chemotherapy, radiation therapy, cause-specific death classification, other cause of death classification, interval between primary and second breast cancer and survival month. We identified 36,006 patients over 20 years old who were first diagnosed with stage I-III primary breast cancer $(\mathrm{BC})$ and received surgical treatment and subsequently developed invasive second primary breast cancer (SBC) from 1992 to 2015 (Fig. 1). The patients might have had different marital statuses when they were first and second diagnosed with breast cancer. Therefore, we defined a change in the marital status of patients with two breast cancer diagnoses as a dynamic change in the marital status of these patients. We excluded patients only diagnosed by autopsy or death certifications, without histological confirmation, or with marital status defined as "Unmarried or Domestic Partner" or "Unknown". Eventually, 33,089 eligible BC patients were included in this study. Marital status at diagnosis was the primary variable, and the participants were classified as married, single, and divorced/separated/widowed (DSW). Therefore, according to the marital status of the primary BC and the SBC, participants were divided into seven groups: Single-Single group $(\mathrm{N}=3306)$, SingleMarried group $(\mathrm{N}=540)$, Single-DSW group $(\mathrm{N}=686)$, Married-Married group $(\mathrm{N}=17,623)$, Married-DSW group $(\mathrm{N}=3043)$, DSW-DSW group $(\mathrm{N}=7333)$, DSW-Married group $(\mathrm{N}=558)$. This study was reviewed and approved by the institutional review board at the Shaoxing Second Hospital, and the data were deidentified; thus, patient consent was not involved.

Statistical analysis. We adopted statistical analytic approaches similar to those used in previous studies ${ }^{15,16}$ that examined the benefit of interventions for BC subsets. Differences in the patient characteristics of SBC were compared between every two groups using Pearson chi-square and T tests for categorical and continuous variables, respectively. Multiple imputation of missing data was performed by a multivariate logistic regression model, and 10 cycles were repeated to produce a final data set. The imputation model included the following variables: patient age (continuous), year of diagnosis, histology type (IDC, ILC and other), T stage, $\mathrm{N}$ stage, $\mathrm{M}$ stage, and chemotherapy (yes or no).

The inverse probability propensity score weighting ${ }^{17}$ was used to balance clinicopathological characteristics between every two groups, and we calculated the propensity scores based on patient age, race, year of diagnosis, insurance, histology type, nuclear grade, T stage, $\mathrm{N}$ stage, M stage, ER status, PR status, surgery, chemotherapy and radiotherapy by a logistic regression model for different marital statuses. Patient characteristics after propensity score adjustment are shown to be balanced in Appendix Tables 1-6 in the Supplement. Then, propensity 
score-weighted log-rank tests and Cox proportional hazards models were conducted to compare OS and BCSS between every two groups, and hazard ratios (HRs) with 95\% confidence intervals (CIs) of BCSS (OS) were calculated from multivariable models that adjusted for year of diagnosis, age, race, year of diagnosis, insurance, histology type, nuclear grade, $\mathrm{T}$ stage, $\mathrm{N}$ stage, $\mathrm{M}$ stage, ER status, PR status, surgery, chemotherapy and radiotherapy. Similar procedures were also performed among subgroups with different ages, and interaction tests were conducted by using a likelihood ratio test to explore whether the survival benefit conferred by marital status varied across subgroups.

To assess the consistency of our findings, we conducted four types of sensitivity analyses. First, we repeated analyses after excluding the missing data containing insurance, ER, and PR status. Then, a proportional subdistribution hazards model was used to calculate the HR of BCSS between every two groups after adjusting for competing events such as death from other causes ${ }^{18}$. Third, the entire analyses were repeated after multiple imputation of unknown data by using random survival forest methodology.

All $P$ values were calculated from 2 -sided tests with a threshold of 0.05 to evaluate the statistical significance of the survival benefit by surgery, and all statistical analyses were performed using R software (version 3.6.1).

Ethical approval. All procedures performed in studies involving human participants were in accordance with the ethical standards of the institutional and/or national research committee and with the 1964 Helsinki declaration and its later amendments or comparable ethical standards. For this type of retrospective study, formal consent is not required. The ethics committee of the Shaoxing Second hospital approved the study.

\section{Results}

Patients' baseline characteristics. In view of the inclusion criteria, this study included 33,089 patients diagnosed with SBC from 1992 to 2015. A flowchart of the study participant selection process is shown in Fig. 1. A summary of the baseline features of the patients in the seven marital status groups is shown in Table 1. Among eligible patients, 3306 patients were grouped into the Single-Single group, 540 patients were grouped into the Single-Married group, 686 patients were grouped into the Single-DSW group, 17,623 patients were grouped into the Married-Married group, 3043 patients were grouped into the Married-DSW group, 7333 patients were grouped into the DSW-DSW group, and 558 patients were grouped into the DSW-Married group. MarriedMarried group patients were more likely to have private insurance ( $80 \%$ vs $72 \%$ and $68 \%)$, to be white ( $83 \%$ vs $79 \%$ and $68 \%$ ) and to have a lower stage tumour than DSW-DSW group patients (Appendix Table 3) and Single-Single group patients (Appendix Table 1). Married-Married group patients were older than Single-Single group patients (median age 62 years vs 57 years; Appendix Table 1) but younger than DSW-DSW group patients (median age 62 years vs 72 years; Appendix Table 3). Compared to Single-Married group patients, Single-Single group patients were older (median age 57 years vs 53 years), less frequently white ( $74 \%$ and $68 \%$ ), and less likely to have private insurance (68\% vs 74\%) (Appendix Table 4). Married-DSW group patients were older (median age 71 years vs 62 years), less likely to have private insurance (77\% vs $80 \%)$, and had a lower stage tumour (68\% vs 73\%) compared to Married-Married group patients (Appendix Table 5). In contrast to DSW-Married group patients, DSW-DSW group patients were older (median age 72 years vs 63 years), less likely to have private insurance ( $72 \%$ vs $78 \%$ ), and had a lower stage tumour (71\% vs $74 \%$ ) (Appendix Table 6).

Effects of marital status on BCSS and OS. A total of 4619 deaths due to breast cancer (14.0\%) and 4796 deaths resulting from other cancers $(14.5 \%)$ were identified in the cohort. The median recurrence time was 64 months (interquartile range, 55 to 74 months). Six models (Model 1, Single-Single group vs Married-Married group; Model 2, DSW-DSW group vs Single-Single group; Model 3, DSW-DSW group vs Married-Married group; Model 4, Single-Single group vs Single-Married group; Model 5, Married-DSW group vs Married-Married group; Model 6, DSW-DSW group vs DSW-Married group) were constructed to examine the association between dynamic changes in marital status and survival benefit. Considering the possible interaction among variables, we conducted multivariate Cox regression analysis adjusting for year of diagnosis, age, race, insurance, histology type, nuclear grade, T stage, $\mathrm{N}$ stage, $\mathrm{M}$ stage, ER status, PR status, surgery, chemotherapy and radiotherapy. After adjusting for clinical factors and considering the propensity score, it was found that compared with the Single-Single group and DSW-DSW group patients, the patients in the Married-Married group were significantly associated with better BCSS (HR 1.13, 95\% CI: 1.03-1.19, $P<0.001$; HR 1.19, 95\% CI: 1.14-1.25, $P<0.001$, respectively) and OS (HR 1.25, 95\% CI: $1.20-1.30, P<0.001$; HR 1.49, 95\% CI: $1.45-1.54, P<0.001$, respectively). In contrast to the DSW-DSW group, the patients in the Single-Single group and the DSW-Married group showed similar BCSS (HR 0.98, 95\% CI: 0.92-1.05, $P=0.660$; HR 1.06, 95\% CI: 0.97-1.15, $P=0.193$, respectively) but better OS (HR 1.14, 95\% CI: 1.09-1.19, $P<0.001$; HR 1.32, 95\% CI: $1.25-1.40, P<0.001$, respectively). Compared with the Single-Single group, the Single-Married group exhibited significantly better BCSS (HR 1.21, 95\% CI: 1.07-1.36, $P=0.003$ ) but showed no difference in OS (HR 1.08, 95\% CI: 0.98-1.18, $P=0.102$ ). In contrast to the Married-DSW group, the Married-Married group showed better BCSS (HR 1.11, 95\% CI: $1.05-1.18, P<0.001$ ) and OS (HR 1.27, 95\% CI: 1.22-1.32, $P<0.001$ ) (Fig. 2).

In addition, the effect of marital status varied depending upon age. In general, the older the $\mathrm{BC}$ patients were, the greater the survival benefit they had. For younger patients aged 20 to 40 years, except for model 5, the other model results suggested that marital status was not significantly associated with BCSS or OS (Fig. 2). However, for older patients ( $\geq 65$ years), the majority of model results proved that marital status was significantly associated with BCSS and OS. In addition, for patients in the ranges of 40-50 years and 50-65 years, the findings were mixed. Some results showed that these patients received a survival benefit from marriage, while other results did not. Furthermore, some results showed that even present marriage would produce negative effects on BCSS and OS (Fig. 3). 


\begin{tabular}{|c|c|c|c|c|c|c|c|c|}
\hline \multirow[b]{3}{*}{ Characteristics } & Total & Single-single & \begin{tabular}{|l|}
$\begin{array}{l}\text { Single- } \\
\text { married }\end{array}$ \\
\end{tabular} & Single-DSW & $\begin{array}{l}\text { Married- } \\
\text { married }\end{array}$ & \begin{tabular}{|l} 
Married- \\
DSW
\end{tabular} & DSW-DSW & $\begin{array}{l}\text { DSW- } \\
\text { married }\end{array}$ \\
\hline & $\mathrm{N}=33, \mathbf{0 8 9}$ & $\mathrm{N}=3306$ & $\mathrm{~N}=540$ & $N=686$ & $N=17,623$ & $\mathrm{~N}=3043$ & $\mathrm{~N}=7333$ & $\mathrm{~N}=558$ \\
\hline & No (\%) & No (\%) & No (\%) & No (\%) & No (\%) & No (\%) & No (\%) & No (\%) \\
\hline \multicolumn{9}{|c|}{ Year of diagnosis } \\
\hline 1992-1997 & $1191(4)$ & $116(4)$ & $8(1)$ & $9(1)$ & $651(4)$ & $46(2)$ & $347(5)$ & $14(3)$ \\
\hline 1998-2003 & $4761(14)$ & $483(15)$ & $67(12)$ & $88(13)$ & $2538(14)$ & $341(11)$ & $1167(16)$ & $77(14)$ \\
\hline 2004-2009 & $10,686(32)$ & $1062(32)$ & $190(35)$ & $218(32)$ & $5707(32)$ & $927(30)$ & $2400(33)$ & $182(33)$ \\
\hline 2010-2015 & $16,451(50)$ & $1645(50)$ & $275(51)$ & $371(54)$ & $8727(50)$ & $1729(57)$ & $3419(47)$ & $285(51)$ \\
\hline \multicolumn{9}{|l|}{ Race } \\
\hline White & $26,502(80)$ & $2235(68)$ & $396(74)$ & $496(72)$ & $14,661(83)$ & $2483(82)$ & $5792(79)$ & $439(79)$ \\
\hline Black & $3777(11)$ & $786(24)$ & $82(15)$ & $160(23)$ & $1249(7)$ & $326(11)$ & $1086(15)$ & $88(16)$ \\
\hline Other & $2800(9)$ & $285(9)$ & $60(11)$ & $30(4)$ & $1711(10)$ & $234(8)$ & $449(6)$ & $31(6)$ \\
\hline Unknown & $10(0)$ & $0(0)$ & $2(0)$ & $0(0)$ & $2(0)$ & $0(0)$ & $6(0)$ & $0(0)$ \\
\hline \multicolumn{9}{|l|}{ Insurance } \\
\hline $\begin{array}{l}\text { Private insur- } \\
\text { ance }\end{array}$ & $17,120(52)$ & $1460(44)$ & $290(54)$ & $318(46)$ & $9674(55)$ & $1761(58)$ & $3308(45)$ & $309(55)$ \\
\hline $\begin{array}{l}\text { Insured/no } \\
\text { specifics }\end{array}$ & $3151(10)$ & $310(9)$ & $53(10)$ & $72(10)$ & $1579(9)$ & $312(10)$ & $764(10)$ & $61(11)$ \\
\hline Any medicaid & $1985(6)$ & 436 (13) & $43(8)$ & $91(13)$ & $544(3)$ & $207(7)$ & $642(9)$ & $22(4)$ \\
\hline Uninsured & $185(1)$ & $42(1)$ & $6(1)$ & $6(1)$ & $81(0)$ & $20(1)$ & $25(0)$ & $5(1)$ \\
\hline Unknown & $10,648(32)$ & $1058(32)$ & $148(27)$ & $199(29)$ & 5745 (33) & $743(24)$ & $2594(35)$ & $161(29)$ \\
\hline \multicolumn{9}{|l|}{ Grade } \\
\hline I & $7133(22)$ & $645(20)$ & $89(16)$ & $142(21)$ & $3824(22)$ & $653(21)$ & $1653(23)$ & $127(23)$ \\
\hline II & $13,418(41)$ & $1288(39)$ & $203(38)$ & $282(41)$ & $7096(40)$ & $1282(42)$ & $3055(42)$ & $212(38)$ \\
\hline III & $10,028(30)$ & $1116(34)$ & $201(37)$ & $213(31)$ & $5369(30)$ & $912(30)$ & $2036(28)$ & $181(32)$ \\
\hline Unknown & $2510(8)$ & $257(8)$ & $47(9)$ & $49(7)$ & $1334(8)$ & $196(6)$ & $589(8)$ & $38(7)$ \\
\hline \multicolumn{9}{|l|}{ Histology } \\
\hline IDC & $22,999(70)$ & $2329(70)$ & $386(71)$ & $458(67)$ & $12,283(70)$ & $2158(71)$ & $4982(68)$ & $403(72)$ \\
\hline ILC & $3528(11)$ & $328(10)$ & $39(7)$ & $76(11)$ & $1910(11)$ & $314(10)$ & $806(11)$ & $55(10)$ \\
\hline Other & $6562(20)$ & $649(20)$ & $115(21)$ & $152(22)$ & 3430 (19) & $571(19)$ & $1545(21)$ & $100(18)$ \\
\hline \multicolumn{9}{|l|}{ AJCC T stage } \\
\hline pT1 & $23,672(72)$ & $2248(68)$ & $346(64)$ & $459(67)$ & $12,923(73)$ & 2077 (68) & $5208(71)$ & $411(74)$ \\
\hline pT2 & $6347(19)$ & $687(21)$ & $125(23)$ & $151(22)$ & $3225(18)$ & $633(21)$ & $1425(19)$ & $101(18)$ \\
\hline pT3 & $1057(3)$ & $125(4)$ & $27(5)$ & $17(2)$ & $509(3)$ & $108(4)$ & $253(3)$ & $18(3)$ \\
\hline pT4 & $861(3)$ & $104(3)$ & $17(3)$ & $26(4)$ & $403(2)$ & $92(3)$ & $208(3)$ & $11(2)$ \\
\hline Any T, Mets & $1152(3)$ & $142(4)$ & $25(5)$ & $33(5)$ & $563(3)$ & $133(4)$ & $239(3)$ & $17(3)$ \\
\hline \multicolumn{9}{|l|}{ AJCC N stage } \\
\hline pN0 & $25,844(78)$ & $2552(77)$ & $411(76)$ & $530(77)$ & $13,757(78)$ & $2381(78)$ & $5775(79)$ & $438(78)$ \\
\hline pN1 & $4832(15)$ & $473(14)$ & $87(16)$ & $95(14)$ & $2621(15)$ & $434(14)$ & $1046(14)$ & $76(14)$ \\
\hline $\mathrm{pN} 2$ & $1217(4)$ & $145(4)$ & $21(4)$ & $23(3)$ & $611(3)$ & $124(4)$ & $268(4)$ & $25(4)$ \\
\hline $\mathrm{pN} 3$ & $1196(4)$ & $136(4)$ & $21(4)$ & $38(6)$ & $634(4)$ & $104(3)$ & $244(3)$ & $19(3)$ \\
\hline \multicolumn{9}{|l|}{ ER } \\
\hline Negative & $6611(20)$ & $714(22)$ & $148(27)$ & $143(21)$ & $3549(20)$ & $610(20)$ & $1344(18)$ & $103(18)$ \\
\hline Positive & $24,129(73)$ & $2319(70)$ & $365(68)$ & $495(72)$ & $12,870(73)$ & $2272(75)$ & $5390(74)$ & $418(75)$ \\
\hline Unknown & $2349(7)$ & $273(8)$ & $27(5)$ & $48(7)$ & $1204(7)$ & $161(5)$ & $599(8)$ & $37(7)$ \\
\hline \multicolumn{9}{|l|}{ PR } \\
\hline Negative & $10,986(33)$ & $1167(35)$ & $201(37)$ & $246(36)$ & $5906(34)$ & $1012(33)$ & $2283(31)$ & $171(31)$ \\
\hline Positive & $19,329(58)$ & $1840(56)$ & $310(57)$ & $382(56)$ & $10,257(58)$ & $1845(61)$ & $4353(59)$ & $342(61)$ \\
\hline Unknown & $2774(8)$ & $299(9)$ & $29(5)$ & $58(8)$ & $1460(8)$ & $186(6)$ & $697(10)$ & $45(8)$ \\
\hline \multicolumn{9}{|l|}{ Surgery } \\
\hline Nonsurgery & $1540(5)$ & $204(6)$ & $31(6)$ & $56(8)$ & $664(4)$ & $179(6)$ & $378(5)$ & $28(5)$ \\
\hline BCS & $12,412(38)$ & $1187(36)$ & $166(31)$ & $237(35)$ & $6419(36)$ & $1168(38)$ & $3011(41)$ & $224(40)$ \\
\hline Mastectomy & $19,120(58)$ & $1913(58)$ & $343(64)$ & $392(57)$ & $10,534(60)$ & $1694(56)$ & $3939(54)$ & $305(55)$ \\
\hline Unknown & $17(0)$ & $2(0)$ & $0(0)$ & $1(0)$ & $6(0)$ & $2(0)$ & $5(0)$ & $1(0)$ \\
\hline \multicolumn{9}{|l|}{ Radiotherapy } \\
\hline No & $22,381(68)$ & $2260(68)$ & 377 (70) & $461(67)$ & $11,761(67)$ & $2063(68)$ & $5091(69)$ & $368(66)$ \\
\hline Yes & $10,073(30)$ & $980(30)$ & $149(28)$ & $210(31)$ & $5517(31)$ & $920(30)$ & $2124(29)$ & $173(31)$ \\
\hline Continued & & & & & & & & \\
\hline
\end{tabular}




\begin{tabular}{|c|c|c|c|c|c|c|c|c|}
\hline \multirow[b]{3}{*}{ Characteristics } & Total & Single-single & $\begin{array}{l}\begin{array}{l}\text { Single- } \\
\text { married }\end{array} \\
\end{array}$ & Single-DSW & $\begin{array}{l}\text { Married- } \\
\text { married }\end{array}$ & $\begin{array}{l}\text { Married- } \\
\text { DSW }\end{array}$ & DSW-DSW & $\begin{array}{l}\text { DSW- } \\
\text { married }\end{array}$ \\
\hline & $N=33,089$ & $\mathrm{~N}=3306$ & $\mathrm{~N}=540$ & $N=686$ & $N=17,623$ & $\mathrm{~N}=3043$ & $\mathrm{~N}=7333$ & $\mathrm{~N}=558$ \\
\hline & No (\%) & No $(\%)$ & No $(\%)$ & No (\%) & No (\%) & No (\%) & No (\%) & No (\%) \\
\hline Unknown & $635(2)$ & $66(2)$ & $14(3)$ & $15(2)$ & $345(2)$ & $60(2)$ & $118(2)$ & $17(3)$ \\
\hline \multicolumn{9}{|l|}{ Chemotherapy } \\
\hline No & $23,373(71)$ & $2183(66)$ & 317 (59) & $471(69)$ & $11,973(68)$ & $2280(75)$ & $5770(79)$ & $379(68)$ \\
\hline Yes & $9716(29)$ & $1123(34)$ & $223(41)$ & $215(31)$ & $5650(32)$ & $763(25)$ & $1563(21)$ & $179(32)$ \\
\hline \multicolumn{9}{|l|}{ Age (years) } \\
\hline $20-40$ & $743(2)$ & $190(6)$ & $53(10)$ & $14(2)$ & $385(2)$ & $43(1)$ & $42(1)$ & $16(3)$ \\
\hline $40-50$ & $3991(12)$ & $654(20)$ & $157(29)$ & $62(9)$ & $2469(14)$ & $221(7)$ & $366(5)$ & $62(11)$ \\
\hline $50-65$ & $11,961(36)$ & $1424(43)$ & $228(42)$ & $268(39)$ & $7326(42)$ & $768(25)$ & $1700(23)$ & $247(44)$ \\
\hline$\geq 65$ & $16,394(50)$ & $1038(31)$ & $102(19)$ & $342(50)$ & $7443(42)$ & $2011(66)$ & $5225(71)$ & $233(42)$ \\
\hline Median (range) & $64(55,74)$ & $57(49,67)$ & $53(45,61)$ & $64(55.25,73)$ & $62(53,71)$ & $71(60,79)$ & $72(63,80)$ & $63(54,70.75)$ \\
\hline \multicolumn{9}{|c|}{ Interval from primary to SBC, median (interquartile range), months } \\
\hline & $73(33,120)$ & $62(26,109)$ & $88(51,128.25)$ & $81(44,129)$ & $72(32,119)$ & $106(66,156)$ & $62(26,106)$ & \begin{tabular}{|l|}
90.5 \\
$(50.25,135)$
\end{tabular} \\
\hline
\end{tabular}

Table 1. Baseline demographic and tumor characteristics of patients according to marital status in SEER database.

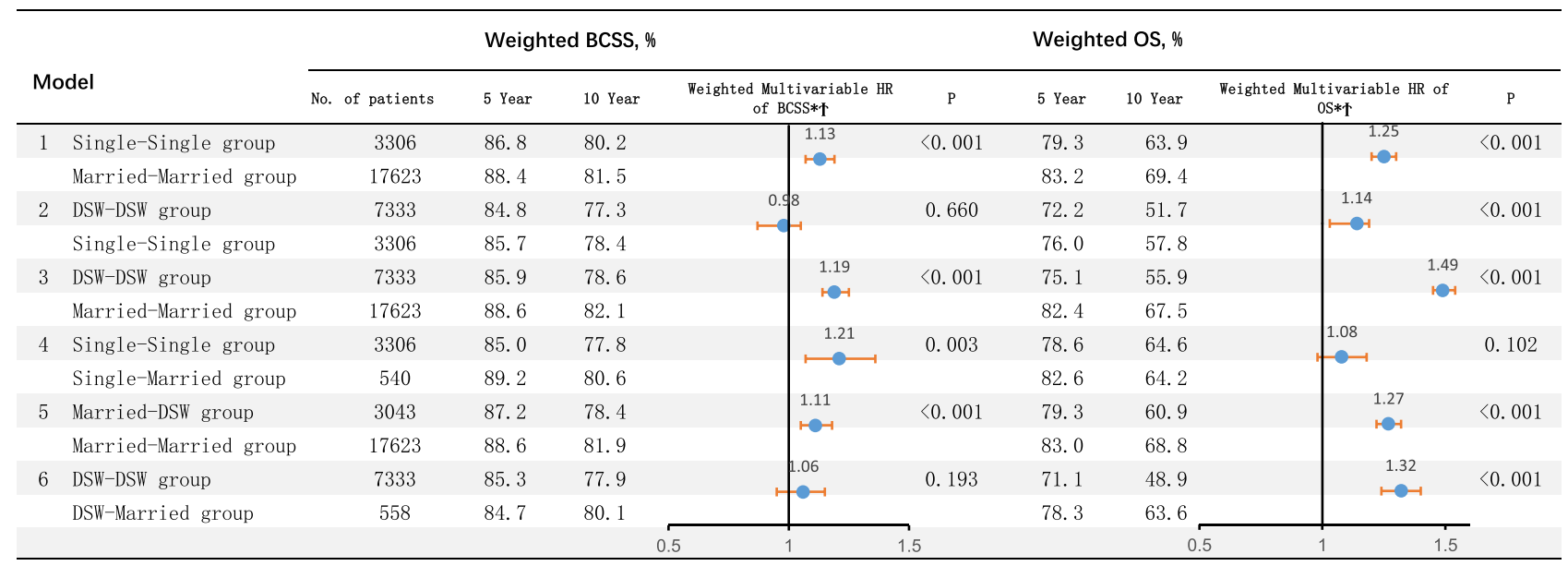

Figure 2. Hazard ratio comparing BCSS and OS according to marital status. $\left(^{*}\right)$ Weighted by inverse propensity score. ( () Multivariate analysis adjusted by year of diagnosis, age, race, year of diagnosis, insurance, histology type, nuclear grade, T stage, $\mathrm{N}$ stage, $\mathrm{M}$ stage, ER status, $\mathrm{PR}$ status, surgery, chemotherapy and radiotherapy. $B C S S$ breast cancer-caused special survival, $O S$ overall survival, $H R$ hazard ratio, $D S W$ divorced/separated/ widowed.

Sensitivity analyses were performed after excluding variables including insurance, ER, and PR status. Repeating the analyses using the proportional subdistribution hazards model and using random survival forest methodology after multiple imputation of unknown data, we observed similar findings.

\section{Discussion}

This SEER analysis is a pioneering study to specifically examine whether dynamic changes in marital status have a significant impact on the survival rate of BC patients. In our study, we observed a remarkably higher risk of death among unmarried SBC patients than among married SBC patients. A noticeable benefit to OS from positive marital status was observed in five models, while that to BCSS occurred in only four models. Previous studies have revealed that a single person has an increased risk of breast cancer, and single patients without a marriage or childbearing history might have worse survival outcomes than DSW patients ${ }^{19}$. In our study, after controlling the possible interactions between variables with multivariate analysis, we discovered that the patients in the Single-Single group displayed no worse BCSS and OS than did those in the DSW-DSW group. This result suggests that the survival benefit of marriage is more due to social and emotional support than to full-term pregnancy.

A growing body of evidence shows that marital status acts as an independent prognostic factor for survival in patients with breast cancer. The influence of marital status on prognosis might be related to tumour stage, receipt of treatment, and social support ${ }^{3,20-22}$. In our study, we found that married patients in each model had a significantly higher prevalence of white race, private insurance and early tumour stage, which would improve 


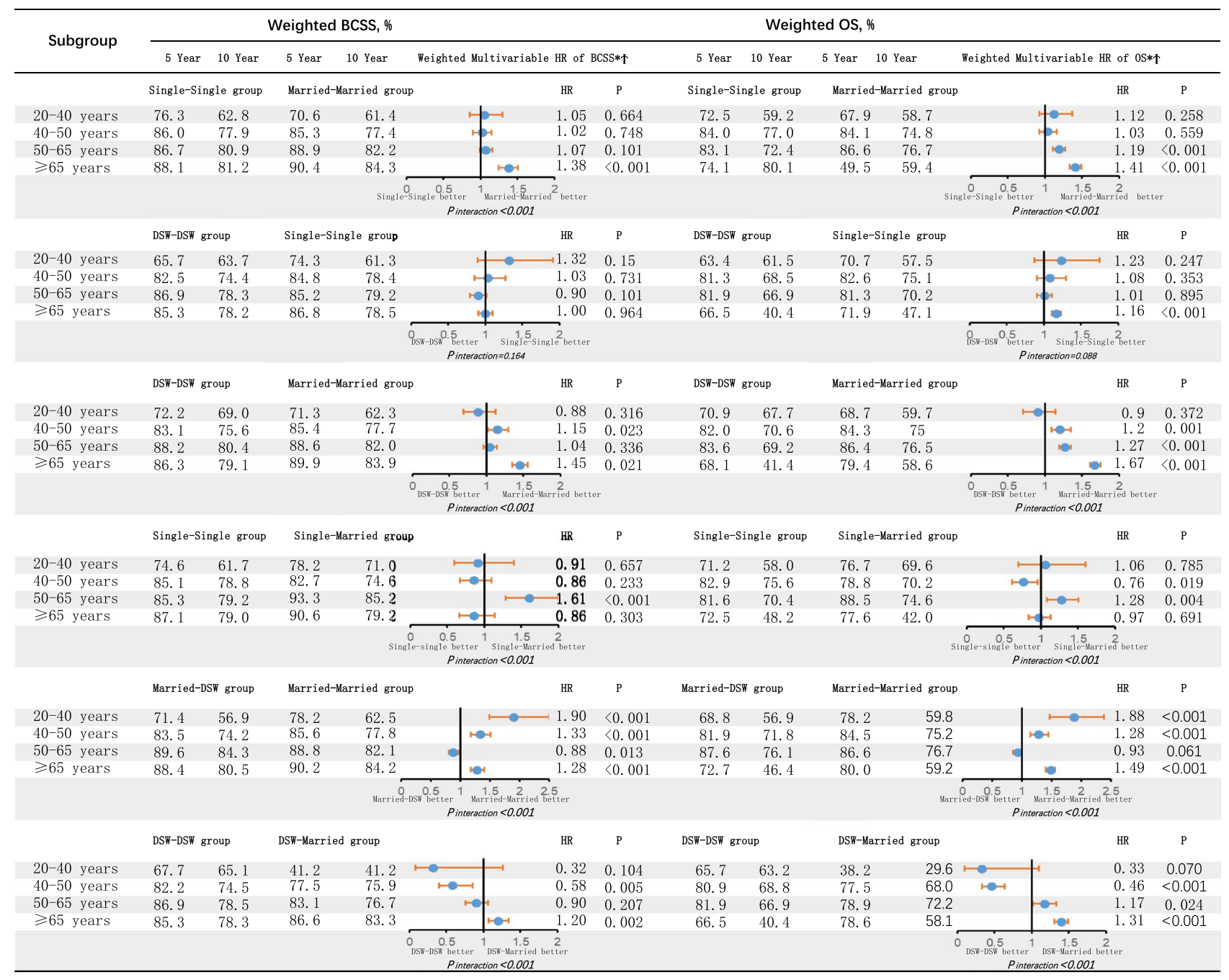

Figure 3. Hazard ratio comparing BCSS and OS according to marital status in different subgroups. $\left(^{*}\right)$ Weighted by inverse propensity score. (T) Multivariate analysis adjusted by year of diagnosis, age, race, year of diagnosis, insurance, histology type, nuclear grade, T stage, $\mathrm{N}$ stage, $\mathrm{M}$ stage, ER status, PR status, surgery, chemotherapy and radiotherapy. BCSS breast cancer-caused special survival, OS overall survival, HR hazard ratio, $D S W$ divorced/separated/widowed.

their prognosis. Women's social relationship influenced by marital status has been proven to impact the choice of mastectomy or breast-conserving surgery whether to receive chemotherapy, and other treatment decisions ${ }^{23-26}$. Our study produced similar results in that the likelihood of receiving radiotherapy, chemotherapy, and surgeries among unmarried groups in each model was lower than that among married groups. Moreover, other studies also showed that a supportive spouse could have a positive effect on his partner's health-seeking behaviour. For instance, investigators have shown that married individuals tend to perform basic and simple healthy behaviours, such as eating more balanced meals and exercising more than unmarried individuals ${ }^{27,28}$. These behaviours can explain why women with a positive marital relationship are less likely to contract breast cancer. In our study, when the patient's marital status changed from single or DSW to married, the median interval time from primary BC to SBC became longer. Although Married-DSW group patients had a longer median interval time than Married-Married group patients, the tumour stage of Married-DSW group patients was more advanced, which suggested that the Married-DSW group patients were not screened for breast cancer in time (Table 1). Cancer screening is a generally effective strategy to detect tumours in the early stage, improve quality of life and reduce costs in health care ${ }^{29-31}$. Hanske et al. claimed that more unfavourable outcomes in unmarried individuals might be linked to a lack of cancer screening in this group ${ }^{32}$. Considering the increasing rate of more advanced stages amongst unmarried patients, it may be hypothesized that cancer screening is less frequent among such patients, which could affect their BCSS and OS.

Furthermore, we discovered an interesting phenomenon in which the survival benefit of marriage was evident in older patients but not in younger patients. This may be because BC treatments are usually more aggressive in young patients, which could result in higher levels of psychological distress, such as chemotherapy-induced menopause and fertility issues. This can impact the couple relationship and, consequently, patient survival ${ }^{33,34}$. Croft et al. highly emphasized that marital status significantly impacted the short-term survival benefit more 
than the long-term survival benefit. This may be because compared with short-term survivors, long-term breast cancer survivors are more likely to be able to resume usual activities and have more time to adjust to their situation. On the other hand, short-term survivors may spend more time at home and seek more assistance from their spouse, partner, or other individuals for daily living activities ${ }^{35}$. These results demonstrate that oncologists and other cancer providers should recognize that unmarried patients (especially short-term survivors), a high-risk group for higher mortality, need more social and psychological support.

Subject to restrictions on our knowledge and research methods, this study has several limitations. The first limitation is that the biases presented in any retrospective study may increase the bias of the results. Second, although assessing marital status at the time of diagnosis of primary BC and SBC is appropriate, we still cannot assess the change in marital status after the breast cancer diagnosis. In addition, it is also important to note that we lack information on the quality of marriage and of support from children and other family members. We also lack information on specific treatments and comorbidities. This information could be a potential confounder in our analyses. Finally, we cannot dismiss the possibility of self-selection in that women who are physically, emotionally, or psychologically healthier may be more likely to marry than their counterparts, which will affect the reliability of the results.

\section{Conclusion}

Our study demonstrated that, regardless of their previous marital status, married patients had a better prognosis than unmarried patients. Meanwhile, single patients obtained better survival outcomes than divorced/separated/ widowed patients. These effects were more significant in older people. For physicians, our results highlight the necessity to proactively provide single and divorced/separated/widowed individuals with appropriate social and psychological support that would benefit them.

Received: 18 June 2020; Accepted: 28 January 2021

Published online: 08 March 2021

\section{References}

1. Ferlay, J. et al. Estimating the global cancer incidence and mortality in 2018: GLOBOCAN sources and methods. Int. J. Cancer 144, 1941-1953 (2019).

2. Gatenby, R. A. \& Gillies, R. J. A microenvironmental model of carcinogenesis. Nat. Rev. Cancer 8, 56-61 (2008).

3. Aizer, A. A. et al. Marital status and survival in patients with cancer. J. Clin. Oncol. 31, 3869-3876 (2013).

4. Pinquart, M. \& Duberstein, P. R. Associations of social networks with cancer mortality: A meta-analysis. Crit. Rev. Oncol. Hematol. 75(2), 122-137 (2010).

5. Gomez, S. L. et al. Effects of marital status and economic resources on survival after cancer: A population-based study. Cancer 122(10), 1618-1625 (2016).

6. Martinez, M. E. et al. Differences in marital status and mortality by race/ethnicity and nativity among California cancer patients. Cancer 122(10), 1570-1578 (2016).

7. Abdollah, F. et al. The effect of marital status on stage and survival of prostate cancer patients treated with radical prostatectomy: A population-based study. Cancer Causes Control 22, 1085-1095 (2011).

8. Osborne, C. et al. The influence of marital status on the stage at diagnosis, treatment, and survival of older women with breast cancer. Breast Cancer Res. Treat. 93, 41-47 (2005).

9. Qiu, M., Yang, D. \& Xu, R. Impact of marital status on survival of gastric adenocarcinoma patients: Results from the Surveillance Epidemiology and End Results (SEER) Database. Sci. Rep. 6, 21098 (2016).

10. Chang, S. M. \& Barker, F. G. II. Marital status, treatment, and survival in patients with glioblastoma multiforme: A populationbased study. Cancer 104, 1975-1984 (2005).

11. ReyesOrtiz, C. A., Freeman, J. L., Kuo, Y. F. \& Goodwin, J. S. The influence of marital status on stage at diagnosis and survival of older persons with melanoma. J. Gerontol Ser. A 62, 892-898 (2007).

12. Goodwin, J. S., Hunt, W. C., Key, C. R. \& Samet, J. M. The effect of marital status on stage, treatment, and survival of cancer patients. JAMA 258, 3125-3130 (1987).

13. Cohen, S. D. et al. Social support and chronic kidney disease: An update. Adv. Chronic Kidney. Dis. 14, 335-344 (2007).

14. Duggan, M. A., Anderson, W. F., Altekruse, S., Penberthy, L. \& Sherman, M. E. The surveillance, epidemiology, and end results (SEER) program and pathology: Toward strengthening the critical relationship. Am. J. Surg. Pathol. 40(12), e94-e102 (2016).

15. Sagara, Y. et al. Survival benefit of breast surgery for low-grade ductal carcinoma in situ: A population-based cohort study. JAMA Surg. 150, 739-745 (2015).

16. Sagara, Y. et al. Patient prognostic score and associations with survival improvement offered by radiotherapy after breast-conserving surgery for ductal carcinoma in situ: A population-based longitudinal cohort study. J. Clin. Oncol. 34, 1190-1196 (2016).

17. Sturmer, T., Rothman, K. J., Avorn, J. \& Glynn, R. J. Treatment effects in the presence of unmeasured confounding: Dealing with observations in the tails of the propensity score distribution a simulation study. Am. J. Epidemiol. 172, 843-854 (2010).

18. Fine, J. P. G. R. A proportional hazards model for the subdistribution of a competing risk. J. Am. Stat. Assoc. 94, 496-509 (1999).

19. Kato, I., Tominaga, S. \& Terao, C. An epidemiological study on marital status and cancer incidence. Jpn. J. Cancer Res. 80, 306-311 (1989).

20. Adekolujo, O. S. et al. Impact of marital status on tumor stage at diagnosis and on survival in male breast cancer. Am. J. Men's Health 11(4), 1190-1199 (2017).

21. Hinyard, L., Wirth, L. S., Clancy, J. M. \& Schwartz, T. The effect of marital status on breast cancer-related outcomes in women under 65: A SEER database analysis. Breast 32, 13-17 (2017).

22. Wang, X., Cao, W., Zheng, C., Hu, W. \& Liu, C. Marital status and survival in patients with rectal cancer: An analysis of the surveillance, epidemiology and end results (SEER) database. Cancer Epidemiol. 54, 119-124 (2018).

23. Hawley, S. T. et al. Decision involvement and receipt of mastectomy among racially and ethnically diverse breast cancer patients. J. Natl. Cancer Inst. 101(19), 1337-1347 (2009).

24. Shelton, R. C. et al. Interpersonal influences and attitudes about adjuvant therapy treatment decisions among non-metastatic breast cancer patients: An examination of differences by age and race/ethnicity in the BQUAL study. Breast Cancer Res. Treat. 137(3), 817-828 (2013).

25. Hershman, D. L. et al. Delay of adjuvant chemotherapy initiation following breast cancer surgery among elderly women. Breast Cancer Res. Treat. 99(3), 313-321 (2006). 
26. Cluze, C. et al. Adjuvant endocrine therapy with tamoxifen in young women with breast cancer: Determinants of interruptions vary over time. Ann. Oncol. 23(4), 882-890 (2012).

27. Meyler, D., Stimpson, J. P. \& Peek, M. K. Health concordance within couples: A systematic review. Soc. Sci. Med. 64, 2297-2310 (2007).

28. Schone, B. S. \& Weinick, R. M. Health-related behaviors and the benefits of marriage for elderly persons. Gerontologist 38, 618-627 (1998).

29. Cohen, J. T., Neumann, P. J. \& Weinstein, M. C. Does preventive care save money? Health economics and the presidential candidates. N. Engl. J. Med. 358(7), 661-663 (2008).

30. Koh, H. K. \& Sebelius, K. G. Promoting prevention under the affordable care act. N. Engl. J. Med. 363(14), 1296-1299 (2010).

31. Maciosek, M. V. et al. Priorities among effective clinical preventive services: Results of a systematic review and analysis. Am. J. Prev. Med. 31, 52-61 (2006).

32. Hanske, J. et al. The influence of marital status on the use of breast, cervical, and colorectal cancer screening. Prev. Med. 89, 140-145 (2016).

33. Mosher, C. E. \& Danoff-Burg, S. A review of age differences in psychological adjustment to breast cancer. J. Psychosoc. Oncol. 23(2-3), 101-114 (2005).

34. Gabriel, C. A. \& Domchek, S. M. Breast cancer in young women. Breast Cancer Res. 12(5), 212 (2010).

35. Croft, L., Sorkin, J. \& Gallicchio, L. Marital status and optimism score among breast cancer survivors. Support. Care Cancer 22(11), 3027-3034 (2014).

\section{Author contributions}

Conception or design: W.D., G.R. Acquisition, analysis, or interpretation of data: W.D., G.R., C.T. Drafting of the manuscript: W.D., Y.L. Critical revision of the manuscript for important intellectual content: W.D., G.R., J.Z., C.T., Z.L. Statistical analysis: W.D., J.Z. Supervision: C.T. All authors have given final approval of the manuscript for submission and publication.

\section{Funding}

Keqiao District Science and Technology Project.

\section{Competing interests}

The authors declare no competing interests.

\section{Additional information}

Supplementary Information The online version contains supplementary material available at https://doi. org/10.1038/s41598-021-84996-y.

Correspondence and requests for materials should be addressed to W.D.

Reprints and permissions information is available at www.nature.com/reprints.

Publisher's note Springer Nature remains neutral with regard to jurisdictional claims in published maps and institutional affiliations.

Open Access This article is licensed under a Creative Commons Attribution 4.0 International License, which permits use, sharing, adaptation, distribution and reproduction in any medium or format, as long as you give appropriate credit to the original author(s) and the source, provide a link to the Creative Commons licence, and indicate if changes were made. The images or other third party material in this article are included in the article's Creative Commons licence, unless indicated otherwise in a credit line to the material. If material is not included in the article's Creative Commons licence and your intended use is not permitted by statutory regulation or exceeds the permitted use, you will need to obtain permission directly from the copyright holder. To view a copy of this licence, visit http://creativecommons.org/licenses/by/4.0/.

(C) The Author(s) 2021 